\title{
Limits on the Radius and a Possible Atmosphere of Charon from Its 1980 Stellar Occultation
}

\author{
J. L.. EI.I.IOT*广 AND L. A. YOUNG* \\ *Department of Earth. Atmospheric, and Planetary Sciences. Massachuseths Institute of Technology, Cambridge, Massachusetts 02139; and \\ †Department of Physics. Massachuse'ts Institute of Technology. Cambridge. Massachusetts 02139 \\ Received February 19. 1990: revised October 26. 1990
}

\begin{abstract}
Stellar occultation data for Charon obtained by Walker (1980, Mon. Not. R. Aston. Soc. 192, 47p-50p) have been fit by a model that includes possible differential refraction by an atmosphere, followed by an abrupt occultation by Charon's limb. We find a lower limit ( $3 \sigma$, where $\sigma=0.8 \mathrm{~km}$ ) on Charon's radius of 601.5 $\mathrm{km}$, which can be used as a constraint for modeling the mutual event data. Although our model fits favor the possibility of a Charonian atmosphere (composition undetermined), the time resolution of the data is insufficient to be certain that an atmosphere has been detected. The data could also be interpreted as being indicative of (i) a slight extinction near Charon or (ii) an as yet unidentified effect, not associated with Charon. For the latter possibilities, we find an upper limit on a Charonian atmosphere of 1 to $57 \mathrm{~cm}$-am, the exact amount depending on the gas assumed to be the major constituent. $\quad \therefore 1991$ Academic Press. Inc.
\end{abstract}

\section{INTRODUCTION}

With the greatest orbital eccentricity and semimajor axis of the known planets, Pluto and its satellite Charon are products of past and present conditions in the region ranging from 30 to $50 \mathrm{AU}$ from the Sun. As such, study of these unique bodies will further our understanding of processes that formed and affected the evolution of this part of the Solar System. According to current ideas, the bulk density of Pluto-Charon, $2.03 \pm 0.04 \mathrm{~g} \mathrm{~cm}^{-3}$ (Tholen and Buic 1989) is high enough to imply that Pluto and Charon formed in the solar nebula, rather than in a protoplanetary nebula with a subsequent escape into a solar orbit (McKinnon and Mueller 1988, Simonelli et al. 1989). Support for this view comes from the fact that Triton-a body that presumably formed near $30 \mathrm{AU}$ in the solar nebula and was subsequently captured by Neptune (Goldreich et al. 1989)-has nearly the same density, $2.05 \pm 0.03 \mathrm{~g} \mathrm{~cm}^{3}$ (Tyler et al. 1989), as PlutoCharon. But how did Pluto and Charon become bound into a "double planet," and what has been their subsequent evolution? Information needed to answer these questions includes their individual densities, which have not yet been determined.

In spite of not knowing their densities, we can hope to make progress in understanding the history of Pluto and Charon by pursuing comparative studies of other propertics, such as their atmospheres, which would be indicative of their composition and processes occurring near their surfaces. Pluto's atmosphere has been probed at high spatial resolution with stellar occultation observations (Elliot et al. 1989, Hubbard et al. 1988), but an atmosphere on Charon has not yet been detected. The constituents of Pluto's atmosphere are $\mathrm{CH}_{4}$ (Buie and Fink 1987) and a heavier gas, probably $\mathrm{N}_{2}$ or $\mathrm{CO}$ (Yelle and Lunine 1989). Since we do not have an accurate value for Pluto's surface radius, which defines the lower boundary of the atmosphere, the equivalent column height of Pluto's atmosphere might be as low as $60 \mathrm{~cm}$-am (Elliot et al. 1989). This value should be compared with the upper limit of 30 $\mathrm{cm}$-am that has been placed on a pure $\mathrm{CH}_{4}$ atmosphere for Charon (Fink and DiSanti 1988). Hence, Charon could have an atmosphere nearly as extensive as Pluto's atmosphere. Furthermore, the ability of Charon to retain an atmosphere could be nearly as great as that of Pluto, if Charon's density turns out to be closer to that of silicates rather than ice.

At present the most sensitive Earth-based method for detecting an atmosphere of a distant body such as Charon is through stellar occultation observations, and in 1980 a stellar occultation by Charon was observed from Sutherland by Walker (1980). With these data he confirmed the existence of Charon as a satellite of Pluto, and his firstorder analysis placed a lower limit of $600 \mathrm{~km}$ on the radius of Charon. This lower limit lies above subsequent determinations of Charon's radius, $593 \pm 20 \mathrm{~km}$ (Tholen and Buie 1988, 1989), from modeling of the series of mutual occultations and eclipses that have just been completed by 


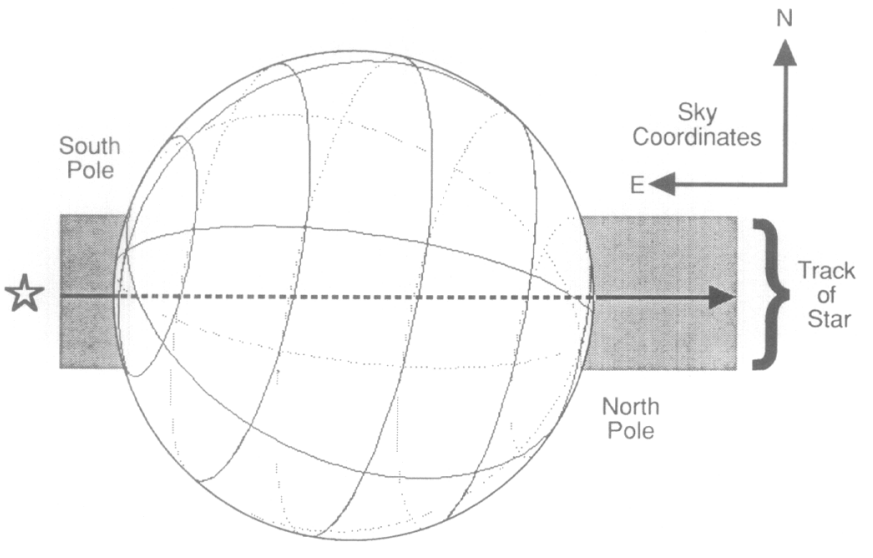

FIG. 1. Occultation geometry for Charon. At the time of the occultation, the south pole of Charon was in sunlight and the north pole in shadow (the north pole is in the direction of the angular momentum vector, opposite to the IAU convention). The highest latitude on the limb was $78^{\circ}$. As illustrated by the shaded area, the exact location of the occultation chord is uncertain.

Pluto and Charon (Binzel et al. 1985). However, Walker's original analysis did not consider the possibility of an atmosphere and included several approximations at the $25-\mathrm{km}$ level, so that his lower limit on the radius is not as rigorous as is now needed for a meaningful comparison with the mutual event results.

Hence, the subject of this note is a reanalysis of Walker's data with two goals: (i) to find a more rigorous lower limit to Charon's radius to compare with the radius from the mutual events and (ii) to place tighter limits on a possible atmosphere of Charon. We carry out this work by fitting a model that includes a possible atmosphere to Walker's occultation light curve.

\section{DATA}

The Charon occultation was observed on April 6, 1980 (UT) by Walker (1980) with the 100-cm telescope of the South African Astronomical Observatory (SAAO) at Sutherland. The aspect of Charon at the time of the occultation is shown in Fig. 1, where we see that the south pole, where the immersion occurred, was exposed to sunlight (the north pole is in the direction of the angular momentum vector, opposite to the IAU convention). AIthough the actual latitudes of the suboccultation points are unknown (since only a single chord was recorded for this occultation and we do not know Charon's surface radius accurately), the maximum possible latitudes of the suboccultation points were $78^{\circ}$ north and south.

Walker used a two-channel photometer with a photon counting system and a B filter. Due to limitations of the data rate that could be recorded, the integration time was set at $2.0 \mathrm{sec}$. Unfortunately. Dr. Walker could not locate his original digital data, but he kindly supplied us with a portion of the data that had been plotted on graph paper that had grid lines at an interval of 100 photons. Although this plot does not include all the previously published data, the larger size of the graph and its grid lines make it substantially more accurate than working from the published figure (Walker 1980). In digitizing these data, one could readily determine whether a point was plotted on a grid line or between two, so that the values were digitized to the nearest 50 photons. Except for two points in the middle of the lower baseline, independent readings of the graph by threc different people gave exactly the same results. Assuming a round-off error in the original plot of a quarter of the grid line spacing (i.e., a point was plotted on a grid line or midway between two), we estimate the rms error between the original digital data and the version used in this analysis as a quarter of a grid line spacing: \pm 25 photons per integration. This can be compared with 60 and 100 photons per integration, the standard deviation expected from Poisson statistics for the signal when the star was occulted and fully visible, respectively. The second channel recorded a comparison star to monitor the sky transparency, which remained constant over the 92 sec of the portion of the data set supplied to us by Walker (not plotted here, see Fig. 2 in Walker 1980).

The upper panel of Fig. 2 shows a plot of Walker's data, where each point represents an individual integration. The upper level signal corresponds to the combined light of the star, Pluto, Charon, and background; the lower level corresponds to the light of only Pluto, Charon, and background.

The main drop in the recorded signal has been attributed to an occultation by Charon (Walker 1980) because the shadow of Charon was predicted to pass less than 0.2 arcsec from SAAO (an amount approximately equal to the error in the astrometry), while the predicted path for the shadow of Pluto was about 0.85 arcsec further south-well off the Earth (Klemola and Elliot 1980). We can now be certain that this was not an occultation by Pluto, since the abrupt nature of the 1980 occultation event is quite different from a more protracted occultation that would be expected for Pluto, duc to its atmosphere (Elliot et al. 1989, Hubbard et al. 1988).

On each end of the fully occulted section of the signal in Fig. 2 is a point that falls approximately midway between full stcllar signal and a full occultation. For a body devoid of an atmosphere, one would interpret both of these points as an average of the unocculted and occulted signals, weighted by the relative portions of the integration interval that the star was occulted and unocculted. However, we note that each point in the "unocculted" region adjacent to each of these "partially occulted" points (indicated by the arrows in Fig. 2) is lower than the unocculted signal. The mean of the 20 points of unocculted 


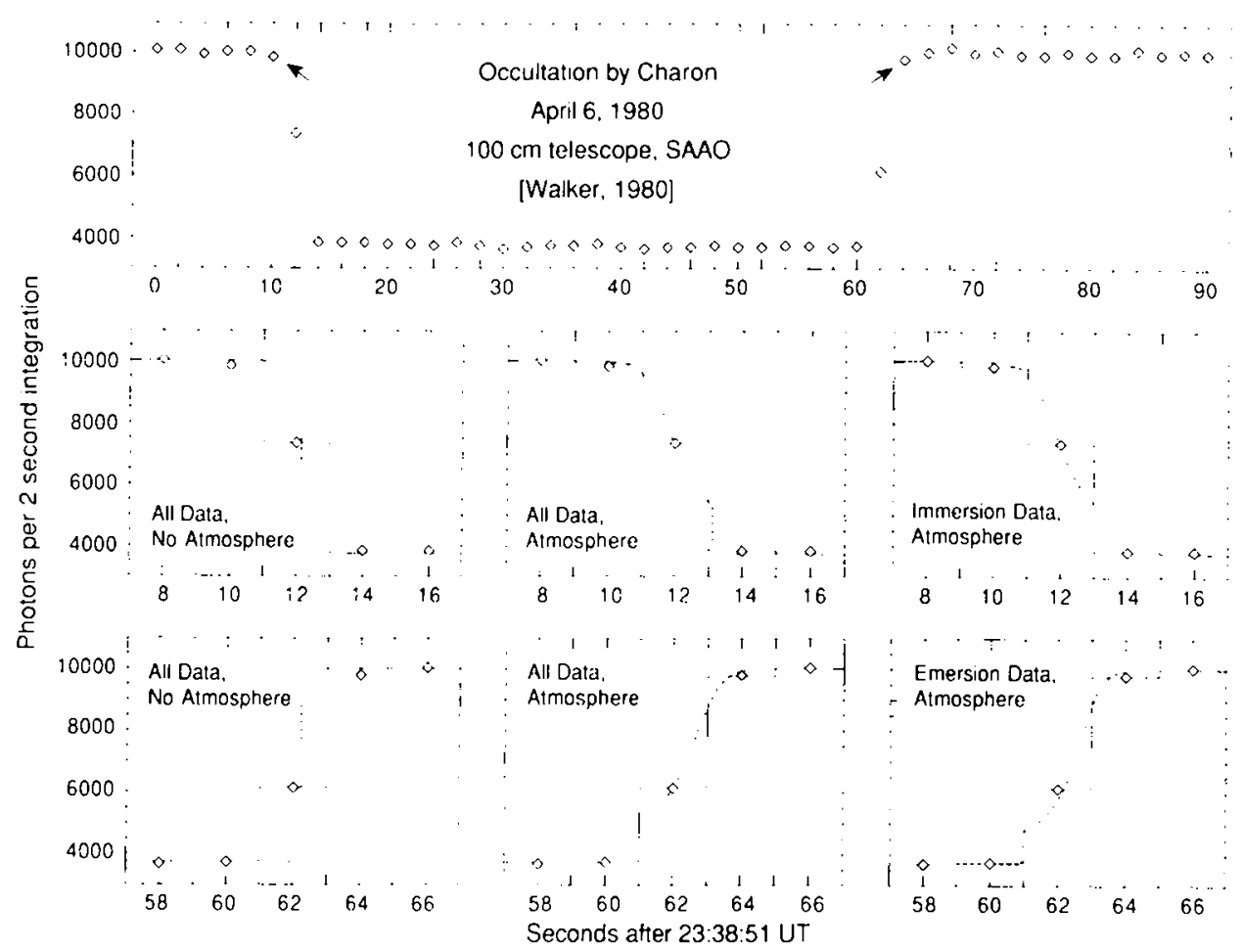

FIG. 2. Charon occultation data and model light curves. The upper panel shows the Charon occultation curve recorded by Walker (1980), in which each point represents a 2.0-sec integration of the combined light of Charon. Pluto, and the occulted star. Note that the two integration intervals adjacent to the main occultation (indicated by the arrows) each have a slightly lower level than the average signal in the unocculted region. One explanation for these lower levels would be a partial occultation of the star. prior to the main occultation by the limb-possibly caused by some type of extinction. or. alternatively. an atmosphere. The middle three panels show model fits to the data, each for a different assumption: (i) Charon has no atmosphere: (ii) Charon has an atmosphere of unconstrained composition: and (iii) Charon has an atmosphere of unconstrained composition (immersion data only). The bottom three panels show the analogous information for emersion. In these plots the dashed lines correspond to the instantaneous value of the light curve, while the solid lines-which represent the model fit to the data-correspond to an integration of the instantaneous light curve over $2.0 \mathrm{sec}$. The "possible atmosphere" assumption yields the better fit to the data, indicating that Charon may have an atmosphere.

signal is 10015 , and the calculated standard deviation $(\sigma)$ for a single point is 96.1 (or 100.I if one assumes Poisson statistics). Choosing the larger standard deviation from Poisson statistics, we find that the pre-immersion point is $1.6 \sigma$ below the average and the post-emersion point is $2.1 \sigma$ below the average of the unocculted signal. The joint probability that both of these two points would be this low due to random noise in the data equals the product the individual probabilities. Approximating the Poisson distribution as a Gaussian, the desired probability is $(1-\operatorname{erf}[1.6 / \sqrt{2}])(1-\operatorname{erf}[2.1 / \sqrt{2}]) / 4 \approx 0.001$.

Another approach to estimating the probability that two points at the edges of the unocculted region are caused by noise is to ask the probability that the two lowest points in the unocculted region, which consists of 20 points in this data set, would occur in the two positions adjacent to the occultation. Standard combinatorial procedures state that the probability that one of the two lowest points would be in the pre-immersion position is $2 / 20$, and the probability that the remaining point would be in the post- emersion position is $1 / 19$. Hence, the probability that both of these events occur-as is the case for the light curve--is $\approx 0.005$. If one examines the larger data set published by Walker (1980), we see two more points (one prior to immersion and one occurring after emersion) that appear to have values similar to the two adjacent to the occultation. However, the unocculted region in the larger data set has 58 data points. Again we ask the probability that two of the four lowest points occur next to the main occultation, and get $4 / 58 \times 3 / 57 \approx 0.004$. Hence we conclude that the chance that two points next to the main occultation are low due to noise is small enough to make it unlikely, but not "impossible."

If these two points are removed, we investigated the possibility that the remaining data in the unocculted region would be unacceptably "quiet." Without these two points, the mean and standard deviation for the remaining 18 points is $10036.1 \pm 74.4$. From the $\chi^{2}$ distribution we find that the probability for the rms noise to be this low for the 18 points is 0.07 - small, but much greater than 
0.005 , the most conservative estimate of the probability that the dip points are only caused by noise (see above).

Since the random noise explanation is highly improbable, we considered other possibilities for the lower values of these points. Without belaboring an essentially unresolvable issue at this point, we find a significant objection to each of these following explanations for the low data points: (i) instrumental (it is difficult for an instrument to "anticipate" a drop in signal prior to immersion); (ii) diffraction (the integration interval with the small drop is centered about 50 Fresnel scales from the limb and would include nearly 4000 fringes; so a $1-2 \%$ average lower signal would not be possible, no matter what the phase of the integration interval and the fringes); (iii) limb irregularities (the features would have to be spires over $25 \mathrm{~km}$ high and no more than a few kilometers wide); (iv) multiple star (the position of the putative companion star would be highly constrained, since it would have to disappear prior to immersion of the main component and reappear following emersion of the main component; alternatively a triple system could be invoked with just the correct properties); and $(v)$ an averaging of the diffraction pattern by the "beam size" defined by the stellar diameter of the occulted star. The diameter of a star with $B=12.88$ (Walker 1980) and spectral type F5, which we estimated from its UBVR colors (Taylor 1979), would subtend $0.31 \mathrm{~km}$ at the Earth-Charon distance. This dimension would average the light curve by only $0.013 \mathrm{sec}$-much smaller than the integration interval and not nearly large enough to produce the observed drop in the light curve. Also, if the stellar diameter were the explanation, the signal in the occulted region would be slightly higher by the same amount the unocculted signal is low.

Since none of the above explanations for the lower values of the two data points adjacent to the main occultation appears particularly likely, we now consider effects associated with Charon. Two come to mind: (i) a slight extinction by material $\sim 40 \mathrm{~km}$ above Charon's surface and (ii) a tenuous atmosphere. In this paper we do not pursue the extinction explanation further, only because we have no readily adaptable model from which to glean more information. However, the atmospheric possibility is amenable to modeling, and we can use the results to constrain the properties of a putative atmosphere. Also, from atmospheric modeling we can place limits on the presence of an atmosphere if one prefers to believe that the two data points are low for reasons other than from the effects of an atmosphere.

\section{LIGHT CURVE ANALYSIS}

In terms of placing a lower limit on Charon's radius, the most conservative interpretation of the low points is that they mark the beginning of an atmospheric occulta- tion, with the remaining part of this ocultation occurring in the time interval that also includes the limb occultation. Hence, we model the occultation data as that for a distant small body with an atmosphere, and we minimize the number of fitted parameters for this small data set by assuming that the atmosphere is isothermal. Models that combine the effects of diffraction by a limb in the presence of a tenuous atmosphere have been constructed by French and Gierasch (1976). However, use of the exact results of a diffraction model is not required for the present case, since the integration time used to record the data corresponds to about 50 times the Fresnel scale $(\sqrt{\lambda D / 2}=$ $0.98 \mathrm{~km}$, where $\lambda$ is the wavelength of light and $D$ is the distance between the occulting body and the observer).

Another effect that we considered including in the model is a central bright spot, produced either by diffraction or refraction. If Charon's limb were perfectly smooth, the bright spot at the Earth would be approximately 0.001 $\mathrm{km}$ in diameter and have a peak intensity equal to that of the unocculted intensity of the star. A rough limb, however, would produce an irregular brightening, much less enhanced, but over a larger region. For example, if the limb were rough on a scale of $1 \mathrm{~km}$, the bright area would be about $1 \mathrm{~km}$ in diameter and reach an average peak intensity of $1.4 \times 10^{-6}$ times the unocculted intensity of the star. A central spot this faint would not be detectable.

A brightening near the center of the shadow could also be created by focusing of the atmosphere all around the planet (Elliot et al. 1977) if the refraction angle of a ray grazing the surface is greater than the angular radius of Charon. Since central brightening is not evident in the data, however, the model used here includes only an occultation by an isothermal atmosphere, followed by a geometric occultation by the limb. As we shall see later, the models that fit the data have a limb occultation occurring before the refraction angle would be large enough to produce a central spot.

Light curves for an occultation by a small body with an atmosphere have been analyzed with a numerical model by Hubbard et al. (1988) and treated as a correction to the result of the large body case by Elliot et al. (1989). We use neither of these approaches here, but instead we use an analytic light-curve model that includes all small-body effects of these models. A detailed derivation of our method will be presented in a later publication; here we give just the equations essential for describing the model. For the atmosphere, we make the usual definition (Chamberlain and Hunten 1987) of the parameter $\lambda$ as the ratio of the gravitational potential of a molecule to $k T$, where $k$ is Boltzmann's constant and $T$ is the gas temperature in $\mathrm{K}$. If $G$ is the gravitational constant, $M_{\mathrm{c}}$ the mass of Charon, $\mu$ the mean molecular weight of the atmosphere, $M_{0}$ the mass of unit atomic weight, and $r$ the distance from the center of Charon, then $\lambda$ is given by 


$$
\lambda=\frac{G M_{\mathrm{c}} \mu M_{0}}{r k T} .
$$

A reference level, $r_{\text {rei }}$, has been chosen so that the number density at the reference level satisfies the following equation, where $N_{1}$ is Loschmidt's number. $\nu_{\mathrm{STP}}$. the refractivity of the atmosphere for standard temperature and pressure, and $D$, the observer-Charon distance.

$$
n_{\mathrm{ref}}=\frac{N_{1} G M_{\mathrm{c}} \mu M_{0}}{k T D \nu_{\mathrm{STP}} \sqrt{2 \pi} \lambda_{\mathrm{rei}}^{\varsigma^{2}}}
$$

With this relation and defining $\lambda_{\text {ref }}-\lambda\left(r_{\text {ref }}\right)$ and $n_{\text {ref }}=$ $n\left(r_{\text {ref }}\right)$. we can write the number density as a function of $r$

$$
n(r)=n_{\text {ref }} \exp \left(\lambda-\lambda_{r e t}\right)
$$

For the purposes of setting a lower limit on Charon's radius, we assumed that the occultation chord was central. Denoting the shadow velocity by $\nu_{\text {shadow }}$, the midtime of the occultation by $t_{\text {mid }}$, and the radius from the center of the shadow by $\rho$, we write

$$
\rho(t)=\nu_{\text {shadou }}\left(t--t_{\text {mud }}\right)
$$

The occultation flux, $\phi(r)$, is expressed as a quantity that has had the background level subtracted and been normalized to the unocculted flux from the star. This flux is affected both by extinction in the atmosphere and differential refraction by the atmosphere. We define the quantity $\tau_{o b}(r)$ as the observed optical depth along the path of the ray with radius of closest approach $r$. The derivative of the refractivity of the atmosphere, $\nu(r)$, is integrated along the ray path within the atmosphere (here $d x$; see Fig. Al in Elliot et al. 1989). By conservation of energy, the flux is enhanced by the ratio of the closest approach radius and the radius of the observer from the center of the occultation shadow

$$
\phi(r)=\frac{r}{\rho(r)} \frac{\left.\exp \mid-\tau_{\mathrm{obs}}(r)\right]}{1+D \frac{d}{d r} \int^{x}, \frac{r}{r^{\prime}} \frac{d \nu\left(r^{\prime}\right)}{d r^{\prime}} d x}
$$

For our atmospheric-model analysis we assumed that there was no extinction. so that the optical depth in Eq. (5) was set to zero.

We implemented our model in Mathematica (Wolfram 1988. Maeder 1990) and fit it to all the data of Fig. 2 for two cases: (i) under the assumption that Charon has no atmosphere and (ii) under the assumption that Charon could have a tenuous atmosphere. In these fits, the shadow velocity of Charon, $\nu_{\text {badow }}$, was determined by adding Charon's motion (from an ephemeris of the mutual events) to the motion of the Pluto-Charon barycenter (calculated from quadratic interpolation of the JPL DE 130 ephemeris tabulated at 15 -min intervals). For the nominal density of Charon $\left(2.0 \mathrm{~cm}^{3}\right)$, the shadow velocity of $24.076 \mathrm{~km} \mathrm{sec} 1$ corresponds to an angular motion of 0.0011365 arsec sec ' (cf. 0.0011 arcsec sec ' was used by Walker). If Charon's density were $3.0 \mathrm{~g} \mathrm{~cm}$ ?. the shadow velocity would have been only $0.003 \mathrm{~km} \mathrm{sec}{ }^{-1}$ less than the value we used. The instantaneous stellar flux was numerically integrated over each 2.0 second data bin with a resulting accuracy of $1 \%$. In the fitting procedure, each point was weighted as the reciprocal of the model value at that point, under the assumption that photon noise is the principal noise source. Results of unweighted fits did not differ significantly from the weighted ones.

The results of the "no atmosphere" and "possible atmosphere" fits are given in the first two columns of Table 1. where the tabulated errors refer to the formal errors from the fits. Model parameters with no error bars in Table 1 were set at fixed values in the fitting procedure. The chi-squares $\left(\chi^{2}\right.$. calculated under the assumption that the random noise in the light curve is photon noise) and the number of degrees of freedom are also given in Table I.

Although the $x^{2}$ from the no atmosphere fit is perfectly acceptable (in fact slightly lower than the average expected for 42 degrees of freedom), we see a dramatic drop in $x^{2}$ from 40.2 to 30.5 when only two free parameters describing the atmosphere are added to the model. To establish the significance of this decrease, we calculated the probability that the reduction could have been caused by the random noise on the light curve. Using the $F$-ratio test as applied to determining the significance of a drop in the sum of squared residuals when more free parameters are added to a model (Dunn and Clark 1974). we find that the probability that two additional, irrelevant free parameters would cause this decrease is only 0.004 . Hence, random noise is an unlikely cause of the decrease in residuals.

As a further test of the possible atmosphere model, we fit the immersion and emersion sections of the light curve separately. The points prior to midoccultation (the first 19 points) were included in the immersion section and the remainder in the emersion section. For these fits we had to fix either the midtime or the surface radius at some value. so we chose to fix the midtime at the value obtained from the fit of the entire light curve.

The results of these individual fits appear in the last two columns of Table I. If the light curve on both sides of the event had an identical shape in the absence of noise and the noise on the light curve were "average," we would expect the individually fitted values of $\lambda_{\text {ref }}$ to have errors about $\sqrt{2}$ times greater than the error for the entire light 
TABLE I

Results of Model Fits

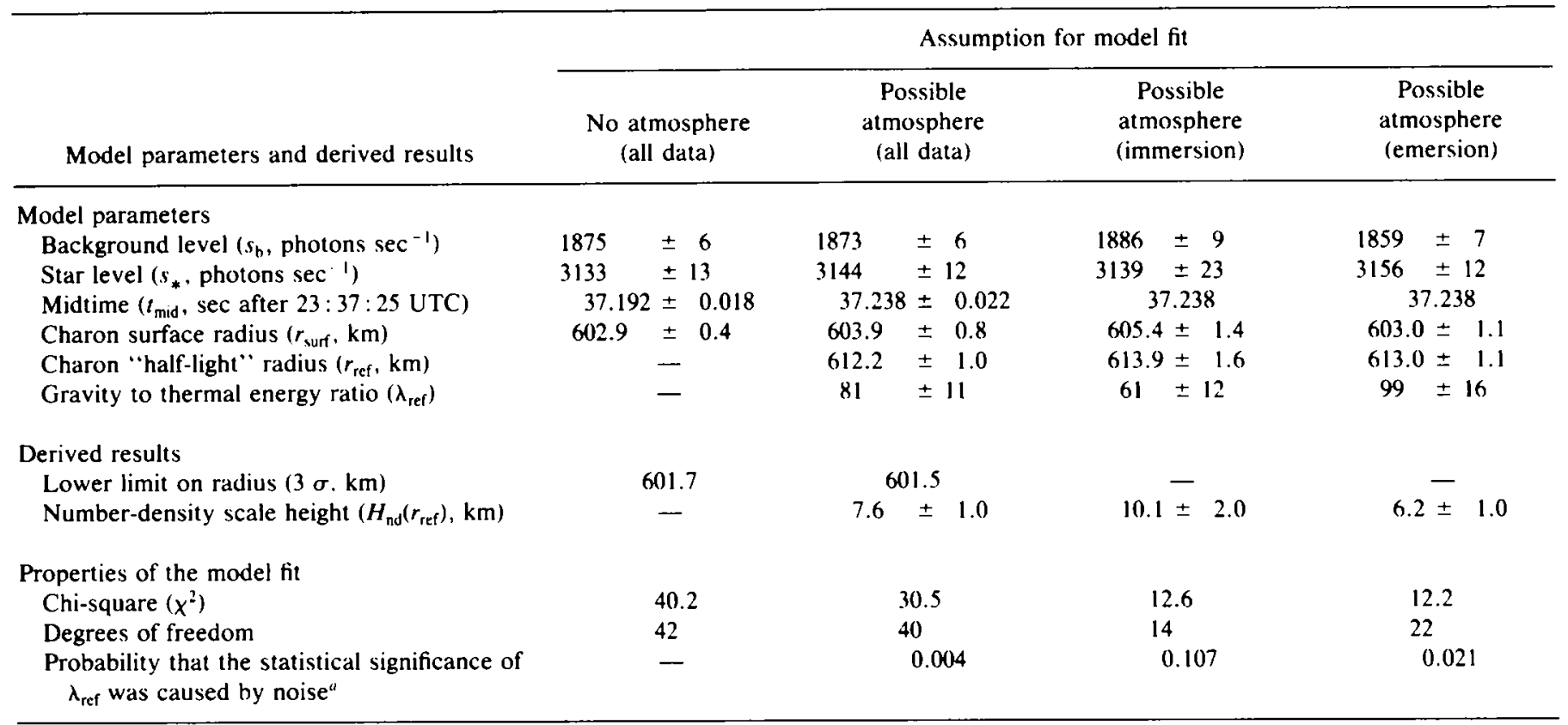

a This was calculated with the $F$-ratio test under the hypothesis that additional two free parameters in the atmosphere fit caused the measured change in $\chi^{2}$ (Dunn and Clark 1974). To perform this test independently for immersion and emersion, additional immersion and emersion fits were performed for the "no atmosphere" case. These had 16 and 24 degrees of freedom, respectively, and they each yielded a $\chi^{2}$ of 17.3 .

curve. Furthermore, we would expect their values to differ from each other by about the root of the sum of their squared standard deviations. The actual difference between the two values of $\lambda_{\text {ref }}$ for these latter two fits is 38 , a value 2.1 times the formal standard deviation of the difference. Hence the "atmosphere" effect appears strongly in both fits, but at a magnitude inconsistent at the $2 \sigma$ level. As we shall discuss in the next section, such a significant difference-caused by a difference between the immersion and emersion atmospheric structure-would be expected from the occultation geometry. The product of the individual probabilities (given in Table I) that noise caused the drop in residuals is 0.002 , of similar magnitude to that found for a fit to the entire curve.

Before proceeding to examine the results of these fits in more detail, we note that the derived lower limit on Charon's radius is nearly the same for both fits. The least stringent lower limit on Charon's surface radius comes from the possible atmosphere case, which corresponds to a $3 \sigma$ lower limit on Charon's radius of $601.5 \mathrm{~km}$. The radius of Charon could be larger if the occultation chord were not central. Our limit should be compared with 593 $\pm 20 \mathrm{~km}$ (Tholen and Buie 1989), where the error has been increased from the published value of $10 \mathrm{~km}$ to account for the uncertainty in the semimajor axis of the Pluto-Charon orbit (Beletic et al. 1989, Tholen and Buie 1988). Our limit would exclude a large portion of the range implied by the mutual event radius and its error, but the difference is not sufficiently large to reject the mutual event solution.

\section{CHARACTERISTICS OF A POSSIBLE CHARONIAN ATMOSPHERE}

We now proceed to infer properties of a possible Charonian atmosphere from either of two assumptions: (i) an atmosphere has been detected, and we can take the results of the light curve fits at face value, or (ii) an atmosphere has not been detected, and one or more effects other than an atmosphere are present in the light curve. Possibilities for nonatmospheric effects were discussed earlier in this paper. However, even if one does not believe that an atmosphere of Charon produced discernable effects in the occultation light curve, the light curve can still be used to place an upper limit on any atmosphere that might surround Charon. We shall now explore the two opposite possibilities for the interpretation of the Charon occultation data: the detection of an atmosphere and the nondetection of an atmosphere.

First, we assume that an atmosphere has been detected. For this case we use the fitted $\lambda_{\text {ref }}$ to solve for the molecular weight of the gas with Eq. (1). However there are two more quantities in that equation-the temperature and mass-that are not well known and for which we must adopt values. For the temperature, Sykes et al. (1987) 
argue for an "isothermal latitude model," in which the surface temperature would be $39 \mathrm{~K}$ for a latitude of $78^{\circ}$. if we use their values for Bond albedo and emissivity assumed for Charon. On the other hand, if the "standard thermal model" is the correct description of Charon. the surface temperature at either of the suboccultation points could be as high as $64 \mathrm{~K}$ if the latitude were as low as $60^{\circ}$. Atmospheric temperatures could be different, of course (probably higher, due to atmospheric absorption of solar radiation). For our present purpose we shall adopt a temperature and error that spans this range: $T=52 \pm 12 \mathrm{~K}$.

To estimate the mass of Charon, it is easiest to think in terms of the more physically diagnostic parameter, the density. We know the system density for Pluto-Charon is near $2.0 \mathrm{~g} \mathrm{~cm}^{3}$. However Charon possesses only about $13 \%$ of the volume of the system. so the system density does not constrain its value, which could reasonably range from 1 to $3 \mathrm{~g} \mathrm{~cm}$ ' (ice to rock). McKinnon (1989) assumes that Pluto-Charon was formed through a collision, and from dynamical arguments he concludes that the maximum density of Charon is $2.3 \mathrm{~g} \mathrm{~cm}^{-3}$. Stern (1988) argues from the lack of detectable volatiles on Charon's surface that its density should not exceed $2.4 \mathrm{~g} \mathrm{~cm}$ ?. For our present purposes, we shall adopt a density and error of $2.0 \pm 0.4 \mathrm{~g} \mathrm{~cm}^{-3}$.

Using these values for temperature and density. and $\lambda_{\text {ret }}$ $=81 \pm 11$ (given in Table I) for the fit to the entire light curve, we find a mean molecular weight of $172 \pm 42$ for the isothermal model. Even with its large uncertainty, this range of molecular weights includes values much higher than the mean molecular weight for the atmosphere of any known solar system body. The molecular weight of $\mathrm{Kr}$ (83.8) lies below the model values, while that of Xe (131.3) is at the lower end. On cosmochemical grounds, a more likely candidate for atmospheric gases on Charon would be $\mathrm{N}_{2}$ - the major constituent of the atmospheres of Titan. Triton. and possibly Pluto (Broadfoot et al. 1989. 1981. Stern and Trafton 1984, Yelle and Lunine 1989). Other possibilities for Pluto (and presumably Charon) would be Ar and $\mathrm{CO}$ (Stern and Trafton 1984. Yelle and Lunine 1989).

The presence of gases this light would be consistent with the data if there were a large atmospheric temperature gradient near the surface of Charon, an effect not included in the model fit to the data. Temperature gradients of a few degrees per kilometer have been observed with radio occultations over the polar caps of Mars (Fjeldbo et al. 1977). In the case of Pluto, the sharp drop in the occultation light curve can be modeled with either an absorbing haze (Elliot et al. 1989) or a large temperature gradient (Eshleman 1989. Hubbard et al. 1990). An isothermal model for the region of the sharp drop in Pluto's occultation curve requires a mean molecular weight about 10 times that required by either the haze or thermal-gradient model (Elliot et al. 1989). If either a strong thermal gradient or extinction prevails on Charon, the molecular weight of its possible atmosphere could even be as low as that of $\mathrm{CH}_{4}$.

When we allow a temperature gradient, we interpret the model scale height from the least-squares fitting as a number density scale height, $H_{\text {nd }}$, which is related to the model parameters through Eq. (6) (Chamberlain and Hunten 1987). We assume that the number-density scale height is constant from the reference level in the atmosphere to the surface (an assumption made for modeling convenience over this small interval).

$$
H_{\mathrm{nd}}\left(r_{\mathrm{ref}}\right)=\frac{r_{\mathrm{ref}}}{\lambda_{\mathrm{ref}}}
$$

In terms of the molecular weight. $\mu$, the temperature. $T$, and the thermal gradient. $d T / d r$, we have the relation, in which the subscript "surf" refers to quantities at the surface, and "ref" refers to quantities at the reference level

$$
H_{\text {nd }}\left(r_{\text {ref }}\right)=H_{\text {nd }}\left(r_{\text {vurf }}\right)=\left[\frac{G M_{\mathrm{c}} \mu M_{0}}{r_{\text {surf }}^{2} k T_{\text {surf }}}+\left(\frac{1}{T} \frac{d T}{d r}\right)_{\text {surt }}\right]^{-1} .
$$

By proper choice of the second term on the righthand side of Eq. (7). one can find a solution for a gas of any molecular weight, but it remains to be seen whether the temperature and its gradient would be acceptable for other criteria. Since large thermal gradients could exist over a surface in vapor-ice equilibrium, we assume that the surface is at the vapor-ice equilibrium temperature for the gas under consideration.

The number density is found with Eq. (2) and extrapolated to the surface with a constant number-density scale height. Then, for a given gas, a surface temperature and thermal gradient is found for which the gas is in vapor-ice equilibrium and its number-density scale height matches that from the occultation light curve with Eq. (6).

Assuming that the occultation data do indicate an atmosphere. one would interpret the difference in $\lambda_{\text {ref }}$ 's for the immersion and emersion light curves as a difference in the atmospheric structure above the two suboccultation points. Since the immersion occultation probed the high latitudes that were in nearly continuous sunlight, while the emersion event occurred at high latitudes that were in nearly continuous shadow (see Fig. 1), the emersion region would be expected to be "colder" (i.e., lower temperature or larger fractional temperature gradient). The difference in fitted $\lambda_{\mathrm{ret}}$ 's is in this sense. In any case, the fact that we find statistically significant $\lambda_{\text {rel }}$ 's on both sides of Charon is consistent with the 
TABLE II

Candidate Gases

\begin{tabular}{|c|c|c|c|c|c|c|c|}
\hline \multirow[b]{2}{*}{ Gas } & \multirow[b]{2}{*}{$\begin{array}{c}\text { Molecular } \\
\text { weight } \\
\text { (amu) }\end{array}$} & \multicolumn{3}{|c|}{$\begin{array}{c}\text { Assumption } \\
\text { Atmosphere detected }\end{array}$} & \multicolumn{2}{|c|}{$\begin{array}{l}\text { Assumption } \\
\text { Atmosphere not detected }\end{array}$} & \multirow[b]{2}{*}{ Conclusions } \\
\hline & & $\begin{array}{l}\text { Column } \\
\text { height } \\
\text { (cm-am) }\end{array}$ & $\begin{array}{c}\text { Suboccultation } \\
\text { surface } \\
\text { temperature } \\
\text { (K) }\end{array}$ & $\begin{array}{c}\text { Near-surface } \\
\text { thermal } \\
\text { gradient } \\
\text { (K km ' }\end{array}$ & $\begin{array}{l}\text { Upper limit on } \\
\text { column height } \\
\text { (cm-am) }\end{array}$ & $\begin{array}{c}\begin{array}{c}\text { Upper limit on } \\
\text { depletion } \\
\text { factor }\end{array} \\
N \text { [atmosphere }] \\
N \text { [cosmic] }\end{array}$ & \\
\hline $\mathrm{CH}_{4}$ & 16.0 & $0.39+0.10$ & $43.5+0.3$ & $5.1 \div 0.8$ & 13. & - & Possible: could be in vapor-ice equilibrium \\
\hline $\mathrm{H}_{2} \mathrm{O}$ & 18.0 & $0.68 \pm 0.17$ & $194.3 \pm 1.1$ & $25.0+3.5$ & 13. & - & Not possible: required surface temperature ton high!! \\
\hline $\mathrm{Ne}$ & 20.2 & $2.6 \div 0.7$ & $10.0=0.1$ & $0.5=0.2$ & 57. & $1.4 \times 10^{9}$ & Possible; could not be in vapor-ice equilibrium \\
\hline$N_{2}$ & 28.0 & $0.58+0.15$ & $30.7+0.2$ & $2.9+0.6$ & 7.7 & - & Possible: vapor-ice equilibrium unlikely \\
\hline $\mathrm{CO}$ & 28.0 & $0.51 \pm 0.13$ & $34.4 \pm 0.2$ & $3.4 \pm 0.7$ & 6.9 & - & Possible, in vapur-ice equilibrium? \\
\hline Ar & 39.9 & $0.61+0.15$ & $35.5+0.2$ & $3.1+0.7$ & 5.0 & $1.8 \times 10^{-7}$ & Possible: in vapor-ice equilibrium? \\
\hline $\mathrm{CO}_{2}$ & 44.0 & $0.38 \pm 0.10$ & $100.0 \pm 0.5$ & $11.4 \pm 1.8$ & 2.1 & - & Not possible: required surface temperature to high! \\
\hline $\mathrm{Kr}$ & 83.8 & $0.40+0.10$ & $49.2+0.3$ & $3.1+1.1$ & 1.5 & $6.0 \times 10^{\circ}$ & Possible: could be in vapor-ice equilibrium \\
\hline$x e$ & 131.3 & $0.24 \pm 0.06$ & $68.1 \pm 0.4$ & $3.7 \pm 1.6$ & 1.0 & $4.7 \times 10^{-4}$ & Possible?: required surface temperature too high? \\
\hline
\end{tabular}

a Fitted light curve parameters interpreted for an atmosphere with a constant number-density scale height $\left(H_{\mathrm{nd}}=7.6 \pm 1.0 \mathrm{~km}\right)$, in vapor-ice equilibrium.

- Upper limits are $3 \mathrm{o}$ and apply for fits of an isothermal model. with subuccultation temperature, $T-52 \pm 12 \mathrm{~K}$ and $\rho_{(\mathrm{charun}}=2.0 \pm 0.4 \mathrm{~g} \mathrm{~cm}$ ?

' For $p_{\text {Churon }}=2.0 \pm 0.4 \mathrm{~g} \mathrm{~cm}$ ?

atmospheric intepretation of the light curve. In the remainder of the paper we shall be using the results of the fit to the entire light curve to derive average properties of the putative atmosphere.

A final quantity of interest for the atmospheric interpretation is the column height of the atmosphere above the surface, $\xi\left(r_{\text {surf }}\right)$, given by the product of the surface number density and the scale height. We denote the surface radius of Charon by $r_{\text {surf }}$.

$$
\xi\left(r_{\text {surf }}\right)=H_{\text {nd }}\left(r_{\text {surf }}\right) n\left(r_{\text {surf }}\right) .
$$

We applied the model just described to various gases. The gases considered and their molecular weights are listed in the first two columns of Table II. Then we used the relation of Eq. (7) and the vapor pressure versus temperature relations (Brown and Ziegler 1980, Washburn 1928 ) to find the implied surface temperature and temperature gradient. These are listed in the fourth and fifth columns of Table II. In the third column of Table II, we give the equivalent column height of the gas, based on Eq. (8). The surface pressure for each of these gases is about 0.1 $\mu$ bar, which might not be sufficient to isothermalize the surface (Trafton and Stern 1983).

Not surprisingly, from this analysis we can rule out $\mathrm{H}_{2} \mathrm{O}$ and $\mathrm{CO}_{2}$ as possibilities, since they would require surface temperatures well in excess of those possible on Charon in order to generate enough vapor pressure to support the amount of gas implied by the light curve. Xenon would be marginally possible in terms of this criterion. Also we can infer that an atmosphere composed of $\mathrm{Ne}$ would not be in vapor-ice equilibrium, since this condition would require a surface temperature lower than could exist on Charon. By the same criterion, vapor-ice equilibrium for an atmosphere of $\mathrm{N}_{2}, \mathrm{CO}$, or Ar would be unlikely.
Now we consider the second view, namely that no atmosphere has been detected. To determine upper limits to the column heights for this case, we performed additional fits to the light curve, one for each gas with the molecular weight fixed and the temperature fixed at $52 \mathrm{~K}$. This isothermal assumption yields larger column heights than those with positive thermal gradients (compare columns 3 and 6 in Table II). Hence these are conservative limits to the column heights, since we believe that no atmosphere around Charon could be extensive enough to have a negative temperature gradient at the surface. From the fitted $\lambda_{\text {ref }}$ and $r_{\text {ref }}$ we then calculated the implied column heights and added 3 standard deviations to the result to obtain the limits given in Table II.

The only conclusions that we draw from the nondetection assumption is that we place stringent upper limits on all gases considered. For example, the limit on $\mathrm{CH}_{4}$ is about three times lower than the spectroscopic limit obtained from mutual event spectra (Fink and DiSanti 1988), although we must remember that these two measurements refer to different ways of detecting atmospheric gas. The spectroscopic measurement refers to an amount integrated over the visible disk, while the occultation refers to the refraction by a column of gas at the limbs (for the present event probably at high latitudes where the temperature would be somewhat lower than average over the visible disk).

\section{SHOULD CHARON HAVE AN ATMOSPHERE?}

Further scrutiny of the possible atmosphere indicated by the occultation data leads us to examine whether an atmosphere of this extent would be possible, based on other information. An atmosphere must have one or more 
sources, and it would escape into space at a rate that depends on the molecular weight of its gases, its temperature at the exobase, and the strength of Charon's gravity.

Potential sources of a Charonian atmosphere include outgassing from the interior and sublimation from the surface-as well as more exotic possibilities, such as capture of gas escaped from Pluto's atmosphere (Whipple et al. 1989) and the sublimation of gas from a small comet impact from the putative Kuiper belt (Duncan et al. 1988. Weissman et al. 1989).

For the noble gases we have computed the amount that could exist in Charon's at mosphere under these optimistic assumptions: (i) all noble gases in the solar nebula were accreted in proportion to their cosmic abundances relative to $\mathrm{Si}$ (Cameron 1982); (ii) Charon is a combination of half "cosmic ice, $" \rho=1.0 \mathrm{~g} \mathrm{~cm} \quad{ }^{3} ; 0 \% \mathrm{Si}$, and "cosmic rock." $\rho=3.0 \mathrm{~g} \mathrm{~cm}{ }^{3}: 17 \% \mathrm{Si}$, (Ozima and Podosek 1983), and (iii) all noble gas within Charon has outgassed into its atmosphere. We can see from Table II that, under these assumptions, there are sufficient amounts of any of these gases to account for the observations. From our results we can place an upper limit on the depletion factor for noble gases. Abundances of other gases depend on the chemical model that one adopts for Charon (Prinn and Fegley 1981). Hence Charon itself could be the source for any of these gases, and only small amounts are needed to model the occultation light curve.

Only an atmosphere of the heaviest gases would be in the Jeans "escape mode. Assuming a density of $2.0 \mathrm{~g} \mathrm{~cm}$ ' for Charon and an exobase temperature of $100 \mathrm{~K}$. we calculate that the observed amount of Xe would escape in $3 \times 10^{7}$ years from an exobase at $950 \mathrm{~km}$ : for $\mathrm{Kr}$. the corresponding values are 650 years and $1900 \mathrm{~km}$. Lighter gases would be in hydrodynamic escape. The mass loss over the age of the Solar System for the lightest gas that we considered. $\mathrm{CH}_{4}$, would deplete only $4 \%$ of Charon's mass, under the assumptions of Trafton et al. (1988).

Another approach to answering the question of whether Charon should have an atmosphere is through comparison with other small Solar System bodies. The most obvious comparison is with Pluto, a body with about twice the diameter of Charon that experiences the same radiation and particle flux from the Sun. Presumably both Pluto and Charon have existed as a binary since the early Solar System. Nevertheless. Pluto has an average surface albedo substantially higher than that of Charon (Tholen and Buie 1989). According to the ideas of Stern et al. (1988). this would imply that the recycling of atmosphere and frost is more prevalent on Pluto. Furthermore. spectra of Charon obtained from the mutual events (by differencing a spectrum of the combined light of Pluto and (haron and a spectrum of Pluto alone) show no $\mathrm{CH}_{4}$ features (to a limit of $30 \mathrm{~cm}$-am) (Fink and DiSanti 1988), but evidence for $\mathrm{H}_{2} \mathrm{O}$ ice (Buic et al. 1987. Marcialis et al. 1987). These spectroscopic observations, however. would not have de- tected $\mathrm{CH}_{4}$ at the upper limit in Table II. Whether these differences between Pluto and Charon result from different initial compositions or are merely superficial differences due to subsequent evolution is not known.

The atmosphere detected on Pluto must have some $\mathrm{CH}_{4}$, accompanied by a heavier gas that is most likely $\mathrm{CO}$ or $\mathrm{N}_{2}$. No matter what its composition, we can infer from the results of Elliot et al. (1989) that the equivalent column height of Pluto's atmosphere could be as low as $60 \mathrm{~cm}$ am-a value somewhat greater than the values or limits for Charon for all gases considered (see Table II). However, the rate of escape (whether Jeans' or hydrodynamic) depends on the parameter $\lambda$, which could be larger at the exobase for Charon than Pluto. If Charon has a density of $3.0 \mathrm{~g} \mathrm{~cm}^{3}$. its surface gravity would be 0.8 that of Pluto's. This, coupled with a higher mean molecular weight and lower exobase temperature would make es. cape from Charon more difficult than from Pluto. This extreme, however, is presumably not the case, since Pluto has more atmosphere than Charon at the present time.

Another comparison for Charon is with Chiron, at a mean heliocentric distance of $13.7 \mathrm{AU}$ and now known to be a comet (Tholen et al. 1988. Meech and Belton 1989. Luu and Jewitt 1990). The radius of Chiron is only about 0.1 that of Charon. Due to the low sublimation rate of $\mathrm{H}_{2} \mathrm{O}$ for the equilibrium temperature at Chiron's solar distance, the coma of Chiron cannot be primarily $\mathrm{H}_{2} \mathrm{O}$. but must be driven by the sublimation of $\mathrm{CO}$ or $\mathrm{N}_{2}$ (Luu and Jewitt 1990). This presents another possibility for an atmosphere of Charon, namely that of a freely escaping. sublimated gas. In this case the density scale height would be large. so that the effect on the occultation data may be extinction by dust being raised above the surface by the escaping gas. rather than the refraction by the gas.

\section{CONCLUSIONS}

Since the lower limit on Charon's radius set by this stellar occultation is insensitive to the presence of an atmosphere in our analysis, our lowest value of $601.5 \mathrm{~km}$ represents a firm lower limit $(3 \sigma)$ on the radius of Charon. This value lies above the radius from the present mutual event model, $593 \pm 20 \mathrm{~km}$ (Tholen and Buie 1988, 1989). This difference between the radii is large enough that future solutions for Charon's radius from the mutual event data would be influenced by the use of our limit as a constraint. The reason for the disparity between the stellar occultation lower limit and the mutual event radius remains to be determined: one possibility would be an underestimate of the semimajor axis of the Pluto-Charon orbit (Beletic et al. 1989), which sets the scale for the dimensions of the system for the mutual event solution.

We believe that Walker's occultation data strongly sugge'st that either extinction by optically thin material near Charon or differential refraction by a tenuous Charonian 
atmosphere caused the starlight to dim by a detectable amount adjacent to the main occultation. This effect occurs in both the immersion and emersion signal. We have pursued the atmospheric possibility more extensively than extinction because a tenuous atmosphere is more amenable to modeling than some arbitrary configuration of particles, and, except for gases with low vapor pressures $\left(\mathrm{CO}_{2}\right.$ and $\left.\mathrm{H}_{2} \mathrm{O}\right)$, there appears no way to rule out the presence of an atmosphere at the levels required to account for the data: less than centimeter-amagat for all gases considered except $\mathrm{Ne}$. Alternatively, if one prefers to interpret the occultation results only as an upper limit, then Charon could not possess an atmosphere of more than a few centimeter-amagats in equivalent column height, for most gases.

Future stellar occultations could resolve this issue of a Charonian atmosphere, at least at the levels discussed in this paper, if any of the potential events identified by Mink et al. (1991) and Dunham et al. (1990) have sufficient signal-to-noise ratio and turn out to be visible from Earth. Also, since the stellar occultation technique is sensitive to minute amounts of any gas, such observations could also be used to search for tenuous atmospheres around other small bodies in the outer Solar System not yet known to have atmospheres- the larger Uranian and Saturnian satellites for example. However, the radii of Charon and most of these satellites as viewed from Earth subtend angles less than a tenth of an arcsec. Accordingly, reliable prediction of these occultations will require greater astrometric accuracy than has routinely been obtained for occultation predictions, and the paths of their shadows crossing the Earth may not include any fixed observatories.

\section{ACKNOWLEDGMENTS}

We thank Alistair Walker for supplying an unpublished version of his data. We also thank Gabriela Canalizo and Linda D'Angelo for digitizing these data, and Eliot Young and Lawrence Wasserman for furnishing the Charon and Pluto emphemerides. Ted Dunham, Richard Baron, Rick Binzel, Alan Stern, Ron Prinn, William Hubbard, Amanda Bosh, and David Tholen (as a referee) provided helpful discussions of issues relevant to this paper. This work was supported, in part, by NASA Grant NAGW-1494 and NSF Grant AST-8906011.

\section{REFERENCES}

Bel.eTIC, J. W., R. M. GoOdy, AND D. J. Tholen 1989. Orbital elements of Charon from speckle interferometry. Ic arus 79, 38-46.

Binzel, R. P., D. J. Tholfen, E. F. Tedesco, B. J. Buratti, and R. M. NELSON 1985. The detection of eclipses in the Pluto-Charon system. Science 228, 1193-1195.

Broadfoot, A. L., S. K. Atreya. J. L. Bertaux, J. E. Bi amont. A. J. Dessler. T. M. Donahue, W. T. Forrester, D. T. Hall, F. Herbert, J. B. Hol Berg. D. M. Hunten, V. A. KrasnopolSKY, S. LINICK, J. I. LUNINE, J. C. MC CONNELI.L. H. W. MOOS. B. R.
Sandei, N. M. Schneider, D. E. Shemansky, G. R. Smith, D. F. Strobel., AND R. V. YelLe 1989. Ultraviolet spectrometer observations of Neptune and Triton. Science 246, 1459-1466.

Broadfoot. A. L., B. R. Sandel, D. E. Shemansky, J. B. Holberg. G. R. S.mith, D. F. Strobel., J. C. McConnel., S. Kumar, D. M. Hunten, S. K. Atreya, T. M. Donahue, H. W. Moos, J. L. BerTACX. J. E. Bl.amont, R. B. POMPHREY. AND S. LiNick 1981. EXtreme ultraviolet observations from Voyager 1 encounter with Saturn. Science 212, 206-211.

Brown. G. N., JR., AND W. T. ZIEGLF.R 1980. Vapor pressure and heats of vaporization and sublimation of liquids and solids of interest in cryogenics below 1-atm pressure. Adv. Cryog. Eng. 25, 662-670.

Buif, M. W., D. P. Cruikshank, L. A. Legofsky, and E. F. Tedesco 1987. Water frost on Charon. Nature 329, 522-523.

BUIE. M. W. . AND U. FINK 1987. Methane absorption variations in the spectrum of Pluto. Lcarus 70, 483-498.

CAMERON, A. G. W. 1982. Elementary and nuclidic abundances in the solar system. In Essays in Nuclear Astrophysics (C. A. Barnes, D. D. Clayton, and D. N. Schramm, Eds.). pp. 23-43. Cambridge Univ. Press, Cambridge.

Chamberi ain. J. W., and D. M. Hunten 1987. Theory of Planetany Atmospheres. Academic Press. Orlando.

Duncan. M., T. Quinn, and S. Tremaine 1988. The origin of shortperiod comets. Astrophys. J. 328, L69-L73.

Dunham, E. W., S. W. Mcdonal.d, and J. L. Elliot 1990. Pluto-Charon stellar occultation candidate search. Bull. Amer. Astron. Soc. 22, 1129.

Dunn, (). J.. A.Nd V. A. CLark 1974. Applied Statistics: Analysis of Variance and Regression. Wiley. New York.

Elliot, J. L.. F. W. Dinham. A. S. Bosh, S. M. Silivan, L. A. Young. L. H. Wasserman, And R. I.. Mil.I.IS 1989. Pluto's atmosphere. Icarus 77, 148-170.

Elliot. J. l., R. G. French, E. Dunham, P. J. Gifrasch, J. Viverka. C. Church, and C. Sagan 1977. Occultation of $\varepsilon$ Geminorum by Mars. II. The structure and extinction of the Martian upper atmosphere. Astrophys. J. 217, 661-679.

Esil f.man, V. R. 1989. Pluto's atmosphere: Models based on refraction, inversion, and vapor-pressure equilibrium. Ic aurs 80, 439-443.

Fink. U., and M. A. DiSanil 1988. The separate spectra of Pluto and its satellite Charon. Astron. J. 95, 229-236.

Fjel dro, G., D. Swetetnam. J. Brenkle, E. Christensen, D. Fari.ess, J. Mehta. B. Sfidfi. W. Michafi. JR.. A. Wallio. AND M. GRossi 1977. Viking radio occultation measurements of the Martian atmosphere and topography: Primary mission coverage. J. Geophys. Re's. 82, 4317-4324.

French. R. G., AND P. J. Gierasch 1976. Diffraction calculation of occultation light curves in the presence of an isothermal atmosphere. Astron. J. 81, 445-451.

Goldreich, P., N. Murray, P. Y. Longaretit, and D. Banfield 1989. Neptune's story. Science 245, 500-504.

Hubrard, W. B., D. M. Hunten. S. W. Dieters, K. M. Hill, and R. D. Warsov 1988. Occultation evidence for an atmosphere on Pluto. Nature 336, 452-4\$4.

HubBard, W. B., R. V. Yelle. AND J. I. Lunine 1990. Nonisothermal Pluto atmosphere models. Icarus 84, 1-11.

Klemola, A. R., anis J. L. Fi.i.iot 1980. No occultation by Pluto on April 6. IAU Circ. No. 3464.

LuU, J. X.. AND D. C. Jewitt 1990. Cometary activity in 2060 Chiron. Astron. J. 100, 913-932.

Mafde.R. R. E. 1990. Programming in Mathematica. Addison-Wesley, Reading. 
Marcial.Is, R. L... G. H. Riekf, and L. A. L.ebofsky 1987. The surface composition of Charon: Tentative identification of water ice. Science' 237, 1349-1351.

MCKininon, W. B. 1989. On the origin of the Pluto-Charon binary. Astrophys. J. 344, L41-L44.

McKinnon, W. B., ANd S. Mufleler 1988. Pluto's structure and composition suggest origin in the solar, not a planetary, nebula. Nature 335, 240-243.

Mrech, K., ANd M. Bei.ton 1989. (2060) Chiron. IAU Circ. Nio. 4770

Mink, S. J., A. R. Klemola. ANd M. W. Bult 1991. Oecultations by Pluto and Charon: 1990-1999. Submitted for publication.

Ozima, M., and F. A. Podosek 1983. Noble Gas Geochemistr. Cambridge Univ. Press. Cambridge.

Prinn, R. G.. ANd B. Fegley Jr. 1981. Kinetic inhibition of $\mathrm{CO}$ and $\mathrm{N}_{2}$ reduction in circumplanetary nebulae: Implications for satellite composition. Astrophys. J. 249, 308-317.

Simonelit., D. P., J. B. Pollack. C. P. Mckay. R. T. Reynol.ds ANid A. L. Summers 1989. The carbon budget in the outer solar nebulia. Icurus 82, 1-35.

StFrn. S. A. 1988. Constraints on Pluto's density and composition lcarks 73, 269-278.

StFrn, S. A., AND L. Trafton 1984. Constraints on bulk composition. seasonal variation, and global dynamics of Pluto's atmosphere. Icarus 57, 231-240.

Stern. S. A.. L. M. Trafton, and G. R. Gladstone 1988. Why is Pluto bright? Implications of the albedo and lightcurve behavior of Pluto. Icarus 75, 485-498.

Sykes. M. V., R. M. CUtri, L. A. Lebofsky, and R. P. BinLEI. 1987. IRAS serendipitous survey observations of Pluto and (haron. Science 237. $1336-1340$.

TAYI OR, G. E. 1979. Possible occultation by Pluto on 1980 April 6. IAU Commission 20. Working Group on Predictions of Ocewlations by Satellites and Minor Planets 15, 1-2.
Tholen, D. J., And M. W. Buif. 1988. Circumstances for Pluto-Charon mutual events in 1989. Astron. J. 96, 1977-1982.

Tholes. D. J.. AND M. W. BuIr 1989. Further analysis of the Pluto-Charon mutual event observations-1989. Bull. Amer. Astr. Soc. 21, 981-982.

Tholen. D. J.. W. K. Hartimann, and D. P. Cruikshank 1988. (2060) Chiron. IAU Cirr. No. 4554.

Trafton, L.. and S. A. Stern 1983. On the global distribution of Pluto's atmosphere. Astrophys. J. 267, 872-881.

Trafton. I... S. A. Stern. And G. R. Giladsione 1988. The Pluto-Charon svstem: The escape of Charon's primordial atmosphere. liarus 74, 108-120.

TYier. G. F.. D. N. Swhetnam. J. D. ANderson. S. E. Borutzki. J. K. Campbel.l. V. R. Eshi.fman. D. L. Gresh, E. M. Gurrol.a. D. P. Hinson, N. Kawashima, E. R. Kursinski, G. S. I.evy, G. F. Lindal. J. R. l.yons, F. A. Marolif, P. A. Rusen, R. A. Simpson, AND G. F. WoOD 1989. Voyager radio science observations of Neptune and Triton. Scicnce 246, 1466-1473.

Wal Kfr. A. R. 1980. An occultation by Charon. Mon. Not. R. Astron. Sor. 192, 47p-50p.

Washburs, E. W. 1928. The vapor pressures of ice and water up to $100^{\circ} \mathrm{C}$. In International Critical Tables of Numerical Data. Physics. (hemistry, and Technology (E. W. Washburn. Ed.). Vol. III, pp. 210. McGraw-Hill. New York.

WEissman. P. R., A. R. Dobrovolskis, and S. A. Stf.rn 1989. Constraints of the population of the Kuiper comet belt based on the low eccentricity of the orbit of Charon. Bull. Amer. Astrom. Sor. 241. 941 .

Whipple. A. L.. L.. M. Trafton, and S. A. Ster. 1989. A gravitational restricted three-body model of Pluto's upper atmosphere. Bull. Amer. Astron. Soc. 21, 982.

Woltram. S. 1988. Mathematica. Addison-Wesley. Reading.

YLI.I.F, R. V.. AND J. I. LuNine 1989. Evidence for a molecule heavier than methane in the atmosphere of Pluto. Nature 339, 288-290. 